\title{
Effects of siRNA Silencing of TUG1 and LCAL6 Long Non-coding RNAs on Patient-derived Xenograft of Non-small Cell Lung Cancer
}

\author{
TIAN FANG ${ }^{1 *}$, HAIRONG HUANG ${ }^{2 *}$, XIAOYOU LI $^{3}$, JING LIAO $^{4}$, ZHIJIAN YANG ${ }^{4,5}$, \\ ROBERT M. HOFFMAN ${ }^{5,6}$, XI CHENG $^{2}$, LEI LIANG $^{1}$, WENJUAN HU ${ }^{1}$ and SHIFENG YUN ${ }^{1}$ \\ ${ }^{1}$ Department of Comparative Medicine, Jinling Hospital, \\ Clinical School of Medical College of Nanjing University, Nanjing, P.R. China; \\ ${ }^{2}$ Department of Cardiothoracic Surgery, Jinling Hospital, \\ Clinical School of Medical College of Nanjing University, Nanjing, P.R. China; \\ ${ }^{3}$ Jiangsu Cancer Hospital, Jiangsu Institute of Cancer Research, \\ Affiliated Cancer Hospital of Nanjing Medical University, Nanjing, P.R. China; \\ ${ }^{4}$ Origin Biosciences Inc., Nanjing, P.R. China; \\ ${ }^{5}$ AntiCancer Inc., San Diego, CA, U.S.A.; \\ ${ }^{6}$ Department of Surgery, University of California, San Diego, CA, U.S.A.
}

\begin{abstract}
Background/Aim: The aim of the present study was to establish a patient-derived xenograft (PDX) mouse model of non-small cell lung cancer (NSCLC) and investigate the antitumor efficacy of silencing of TUG1 and LCAL6 long noncoding RNA in the PDX model. Materials and Methods: PDXs were established by subcutaneously implanting NSCLC surgical tumor fragments into immunodeficient mice. PDX characterization was performed by histopathological, immunohistochemical and real-time polymerase chain reaction (RT-PCR) analyses for NSCLC subtype-specific markers and expression of LCAL6 and TUG1. Anti-tumor efficacy of siRNA silencing of TUG1 and LCAL6 was also investigated in the PDX model. The effect of TUG1 and LCAL6 silencing on protein expression of proliferation marker Ki67 and HOX-gene family HOXB7 in the tumors was assessed by immunohistochemical staining and Western blotting. Results: Establishment of NSCLC PDX models resulted in 9 of 26 cases (34.6\%). Lung squamous cell carcinomas (SCC) had a higher engraftment rate (58.3\%) than lung adenocarcinomas $(A D C)(18.2 \%)(p<0.05)$. Comparative analysis indicated these established PDX models
\end{abstract}

\footnotetext{
*These Authors contributed equally to this work.

Correspondence to: Shifeng Yun, Department of Comparative Medicine, Jinling Hospital, Clinical School of Medical College of Nanjing University, Nanjing, P.R. China. Tel: +86 2580860302, e-mail: yunshifeng1@163.com
}

Key Words: siRNA, patient-derived xenograft, lung cancer. of NSCLC closely resembled the original tumors with regard to NSCLC subtype-specific markers TTF-1, napsin A, p63 and expression of LCAL6 and TUG1. The tumor volume and weight were significantly reduced in the TUG1-silenced group as compared to the control group $(p<0.05)$. However, no significant tumor growth inhibition was found in the LCAL6-silenced group $(p>0.05)$. Expression of both TUG1and LCAL6 was reduced by siRNA treatment. Expression of Ki67 and HOXB7 was significantly suppressed in both the TUG1-and LCAL6-silenced groups compared to the control group $(p<0.01)$. The TUG1silenced group showed more reduced Ki67 expression than the LCAL6-silenced group $(p<0.05)$. Conclusion: PDX NSCLC models were established with a high degree of similarity with the original tumor with regard to histological, immunohistochemical features and RNA expression of TUG1 and LCAL6. Silencing of TUG1 inhibited both tumor growth and expression of the proliferation marker Ki67 and HOX-gene family HOXB7 in the PDX model of NSCLC.

Non-small cell lung cancer (NSCLC) accounts for over $80 \%$ of lung cancer deaths $(1,2)$. The prognosis of NSCLC is still poor (3-5).

Taurine up-regulated gene 1 (TUG1) is induced by taurine. TUG1 is over-expressed and can promote cell proliferation in some cancers (6-9). Lung-cancer-associated transcript 6 (LCAL6) is up-regulated and correlated with tumor progression in lung adenocarcinoma (10). Silencing of TUG1 and LCAL6 inhibited tumor growth and metastasis in some cancer models $(10,11)$. However, the understanding for the roles of TUG1 and LCAL6 in lung cancer is still very limited. 
In the present study, we established a panel of patient-derived xenografts (PDXs) of NSCLC (12-18) and characterized these xenograft models for expression of TUG1 and LCAL6. Furthermore, the anti-tumor efficacy of silencing of TUG1 and LCAL6 was investigated in a PDX model of NSCLC.

\section{Materials and Methods}

Patient tumor samples. A total of 26 primary NSCLC tissues were collected from patients who had undergone surgery at the Jinling Hospital (the Second Affiliated Hospital of Nanjing Medicine University, Nanjing, China) between 2016 and 2017. Tumors were classified as NSCLC based on the World Health Organization (WHO) criteria upon examination by licensed pathologists. None of the patients received chemotherapy or radiotherapy before surgery. Written informed consent was obtained from each patient and the study was approved by the Institutional Review Board of Nanjing Medical University.

Animals. Animal experiments were conducted in accordance with the Institute for Laboratory Animal Research Guide for the Care and Use of Laboratory Animals and approved by the Animal Center of Jinling Hospital (Nanjing, P.R. China). BALB/C male nude mice, 6-8 weeks, 20-25 g, were purchased from the Beijing Vital River Laboratory and Animal Technology Co., Ltd. (Beijing, P.R. China). All mice were maintained in a HEPA-filtered environment at 24$25^{\circ} \mathrm{C}$ and humidity was maintained at 50-60\%. All animals were fed with in autoclaved laboratory rodent diet.

Tumor engraftment and PDX maintenance. Immediately after surgery, fresh tumor tissues were placed in RPMI 1640 culture medium (Invitrogen, Carlsbad, CA, USA) supplemented with $1 \mathrm{mmol} / 1 \mathrm{~L}-$ glutamine, $10 \%$ fetal bovine serum (FCS) (Life Technologies Inc., Burlington, Canada) and antibiotics (100 $\mathrm{U} / \mathrm{ml}$ penicillin $\mathrm{G}$ and 100 $\mu \mathrm{g} / \mathrm{ml}$ streptomycin) and transferred to the animal facilities in Jinling Hospital within 1-2 h after resection. Necrotic tissues were removed and viable tissues were cut with scissors and minced into $2-3 \mathrm{~mm}^{3}$ pieces. Animals were anesthetized by injection of $0.02 \mathrm{ml}$ of a solution comprising 50\% ketamine, 38\% xylazine, and $12 \%$ acepromazine maleate. Tumor fragments were subcutaneously engrafted on the flank of nude mice. The exponentially-growing tumors were harvested and passaged subcutaneously to other mice. After 3-5 consecutive passages, the xenografts were stabilized and subsequently subjected to model characterization. Fresh xenograft tumor fragments were cryopreserved in a medium containing $10 \%$ DMSO in liquid nitrogen, using standard techniques, for future model recovery. All surgical procedures and animal manipulations were conducted under HEPA-filtered laminar-flow hoods.

Histology and immunohistochemistry. For histological examination, tumor tissue specimens were fixed in $4 \%$ formalin, embedded in paraffin, sectioned at $4 \mu \mathrm{m}$ and stained with hematoxylin and eosin (H\&E). Tumor histology was evaluated by a pathologist who was blinded to the experimental groups. For immunohistochemical analysis, formalin-fixed, paraffin-embedded tumor tissues were sectioned at $4 \mu \mathrm{m}$ thickness. All sections were deparaffinized using $100 \%$ xylene, dehydrated with an ethanol gradient. Antigen retrieval was performed by autoclaving $\left(120^{\circ} \mathrm{C}\right.$ for $2 \mathrm{~min}$ in $1 \mathrm{mmol} / \mathrm{l} \mathrm{EDTA}$, $\mathrm{pH}$ 8.0). Incubation with primary antibodies against thyroid
Table I. In vivo tumor take rate of primary NSCLCs according to clinicopathological characteristics.

\begin{tabular}{|c|c|c|c|}
\hline Parameters & Class & $\begin{array}{c}\text { Tumor take } \\
\text { rate }(\%)\end{array}$ & $\mathrm{p}$-Value \\
\hline Age & $\begin{array}{l}<60 \text { years } \\
\geq 60 \text { years }\end{array}$ & $\begin{array}{c}3 / 7(42.8) \\
6 / 19(31.5)\end{array}$ & 0.592 \\
\hline Sex & $\begin{array}{l}\text { Male } \\
\text { Female }\end{array}$ & $\begin{array}{l}8 / 23(35) \\
1 / 3(33.3)\end{array}$ & 0.960 \\
\hline Smoking status & $\begin{array}{l}\text { Ever } \\
\text { Never }\end{array}$ & $\begin{array}{c}6 / 17(35.2) \\
3 / 9(33.3)\end{array}$ & 0.920 \\
\hline Histologic subtype & $\begin{array}{l}\mathrm{ADC}^{\mathrm{a}} \\
\mathrm{SCC}^{\mathrm{b}} \\
\text { Others }\end{array}$ & $\begin{array}{c}2 / 11(18.2) \\
7 / 12(58.3) \\
0 / 3(0)\end{array}$ & $0.049 *$ \\
\hline Tumor size & $\begin{array}{l}<3 \mathrm{~cm} \\
\geq 3 \mathrm{~cm}\end{array}$ & $\begin{array}{c}3 / 8(37.5) \\
6 / 18(33.3)\end{array}$ & 0.837 \\
\hline Overall tumor stage & $\begin{array}{c}\mathrm{I} / \mathrm{II} \\
\mathrm{III} / \mathrm{IV}\end{array}$ & $\begin{array}{c}3 / 13(23) \\
6 / 13(46.1)\end{array}$ & 0.216 \\
\hline
\end{tabular}

aADC: Adenocarcinoma; bSCC: squamous cell carcinomas, ${ }^{*} p<0.05$.

transcription factor-1 (TTF-1) (1:100, ab40880, Abcam, Cambridge, U.K.); napsin-A (1:250, ab133249, Abcam); p63 (1:100, ab53039, Abcam; Ki67 (1:300, VP-RM04, Vector Laboratories, Burlingame, CA, USA); and homeobox B7 (HOXB7) (1:100, ab196007, Abcam) was performed overnight at $4^{\circ} \mathrm{C}$. After washing with $\mathrm{pH} 7.4$ phosphate-buffered saline (PBS), the sections were then incubated with a secondary antibody (Dako REAL EnVision Detection System, Dako, Stockport, UK) for $30 \mathrm{~min}$ at room temperature. Color development was performed with 3, 3'-diaminobenzidine (DAB). Nuclei were lightly counter stained with hematoxylin. The slides were viewed at $\times 400$ magnification and positive cells were recognized by the appearance of brown staining. Expression levels were quantified by counting the number of positive cells in at least 3 fields/sample and expressing the results as percentage of the total number of cells in the same field.

Treatment. siRNA targeting TUG1 (siTUG1) (sense 5'-CUACA ACUAUCUUCCUUUACCACCG-3'; antisense 5'-CGGUGGU AAAGGAAGAUAGUUGUAGCA-3') and LCAL6 (siLCAL6) (sense 5'-CCUGCAGAUAGAUGCUCAAGU-3'; antisense 5'UUGAGCAUCUAUCUGCAGGUG-3') was obtained from Gene Pharma (Shanghai, P.R. China). Treatments were initiated when the average tumor size reached $100 \mathrm{~mm}^{3}$. The mice with tumors were randomly divided into three groups of 6 mice each: Group 1 served as normal control and received saline only. Group 2 (siTUG1) received siTUG1 treatment at $1.5 \mathrm{mg} / \mathrm{kg} /$ dose. Group 3 (siLCAL6) received siLCAL6 treatment at $1.5 \mathrm{mg} / \mathrm{kg} / \mathrm{dose}$. All treatments were administered by intraperitoneal injection (ip) twice per week for 2 weeks. Tumor growth was measured twice a week with calipers. Tumor volume was calculated using the formula $\left(\mathrm{L} \times \mathrm{W}^{2}\right) \times 1 / 2$, where $\mathrm{W}$ and $\mathrm{L}$ represent the perpendicular minor dimension and major dimension, respectively. All animals were sacrificed four weeks after treatment initiation. At autopsy the tumors were removed and weighed.

Real-time polymerase chain reaction (RT-PCR). Total RNA was extracted from tissues with trizol reagent (Life Technologies, Scotland, UK) according to the manufacturer's protocol. Total RNA 


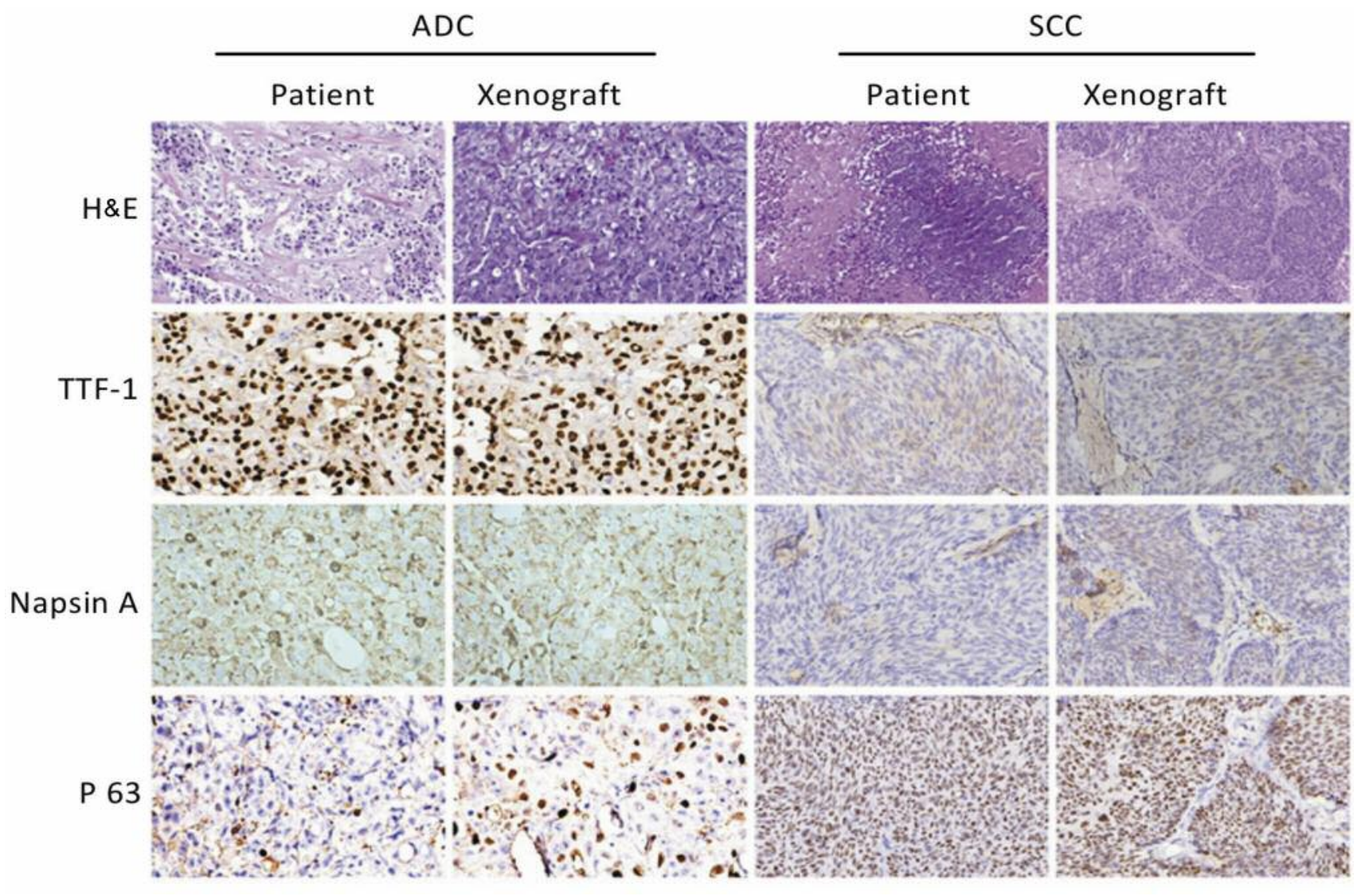

Figure 1. Preservation of parental NSCLC's characteristics in PDX. Representative images of hematoxylin and eosin (H\&E) and immunohistochemically stained NSCLC subtype-specific markers TTF-1, napsin A and p63 in patient tumors and corresponding PDX models $(\times 400$ magnification). ADC: Adenocarcinoma; SCC: squamous cell cancer.

Table II. Expression of NSCLC histologic subtype-specific markers in patient tumor and corresponding PDX.

\begin{tabular}{|c|c|c|c|c|c|c|}
\hline & \multicolumn{3}{|c|}{$\mathrm{ADC}$} & \multicolumn{3}{|c|}{ SCC } \\
\hline & TTF-1 & Napsin A & p63 & TTF-1 & Napsin A & p63 \\
\hline Patient tumor & $84.6 \pm 6.92 \%$ & $5.2 \pm 3.54 \%$ & 0 & 0 & 0 & $82 \pm 8.19 \%$ \\
\hline PDX & $83.6 \pm 8.02 \%$ & $9.8 \pm 0.55 \%$ & $8.3 \pm 1.26 \%$ & 0 & 0 & $95.5 \pm 5.51 \%$ \\
\hline
\end{tabular}

$(1.5 \mu \mathrm{g})$ was reverse transcribed in a final volume of $20 \mu \mathrm{l}$ using random primers under standard conditions using the PrimeScript RT Master Mix (Takara, Cat: RR036A). After the RT reaction, quantitative RT-PCR was performed using the SYBR Select Master Mix (cat: 4472908, Applied Biosystems, Foster City, CA, USA) with $0.5 \mu$ l complementary DNA (cDNA) on an ABI 7300 system (Applied Biosystems, Foster City, CA, USA) according to the manufacturer's instructions. The primer sequences were as follows: GAPDH (sense, 5'-CGCTCTCTGCTCCTCCTGTTC-3', and antisense, 5'-ATCCGTTGACTCCGACCTTCAC-3'); TUG1(sense, 5'-TAGCAGTTCCCCAATCCTTG-3', and antisense, 5'-CACAA
ATTCCCATCATTCCC-3'); LCAL6 (sense, 5'-GTCCTCAAGAC CACAACCCT-3', and antisense, 5'-ACCATCCACGAGTCAG TTCA-3'). The RT-PCR reaction included an initial denaturation step at $95^{\circ} \mathrm{C}$ for $10 \mathrm{~min}$, followed by 40 cycles of $92^{\circ} \mathrm{C}$ for $15 \mathrm{~s}$ and $60^{\circ} \mathrm{C}$ for $1 \mathrm{~min}$. The $\mathrm{Ct}$ value for each sample was calculated with the $\Delta \Delta \mathrm{Ct}$ method, and expression-fold changes (tumor vs. normal) were calculated using $2^{-\Delta \Delta C T}$ methods.

Western blotting. Protein from tumor tissue was extracted with RIPA buffer (Roche Diagnostics, Indianapolis, IN, USA). Total proteins were separated by sodium dodecylsulfate-polyacrylamide gel 

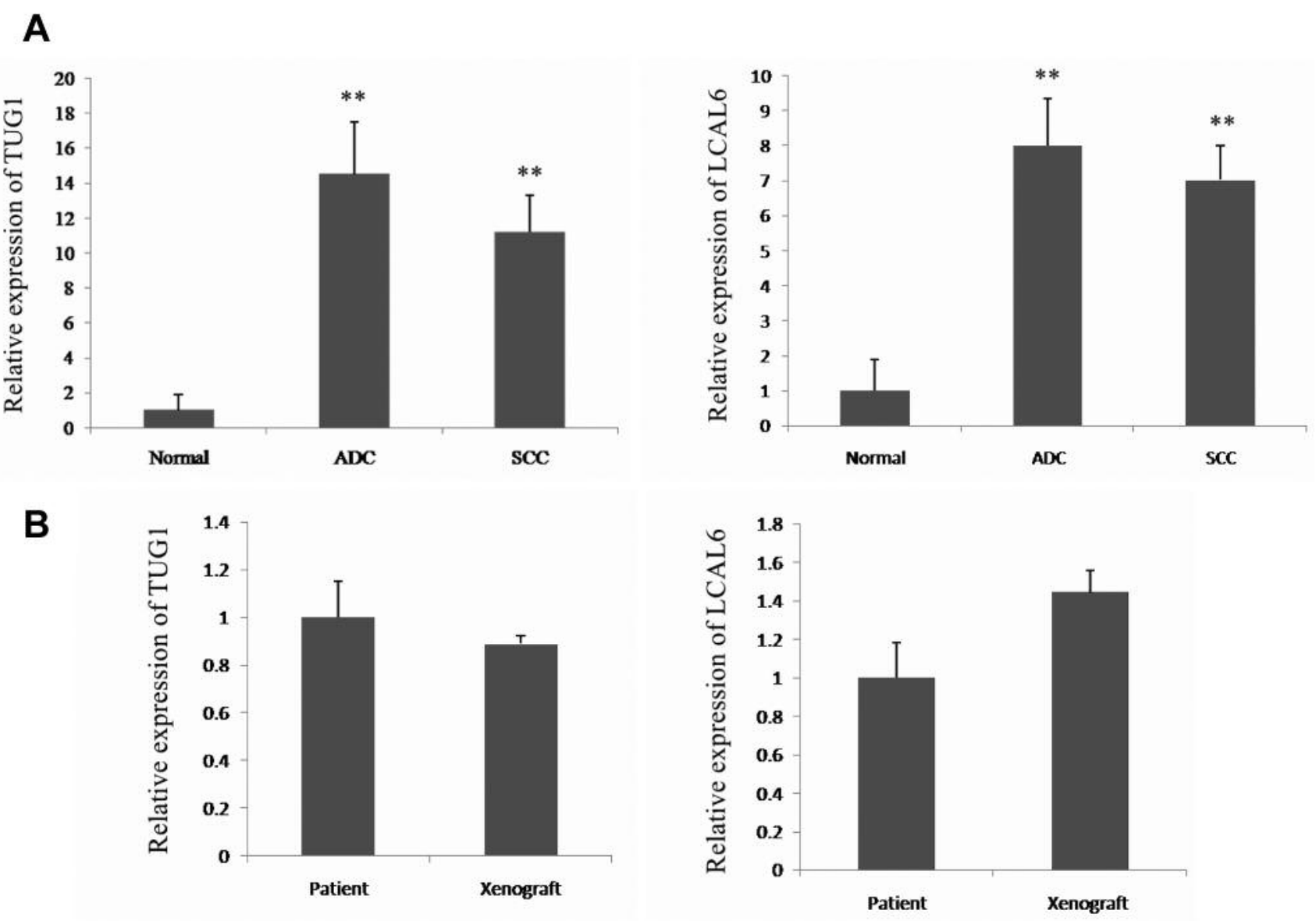

Figure 2. Expression of TUG1 and LCAL6 long non-coding RNAs in patient tumors and corresponding PDXs. TUG1 and LCAL6 expression was detected by RT-PCR. A. Comparison of TUG1 and LCAL6 expression between normal lung tissues and patient tumors. ADC: Adenocarcinoma; SCC: squamous cell cancer. ${ }^{* *} p<0.01$, when compared with normal lung tissues. B. Comparison of TUG1 and LCAL6 expression between patient tumor and PDX.
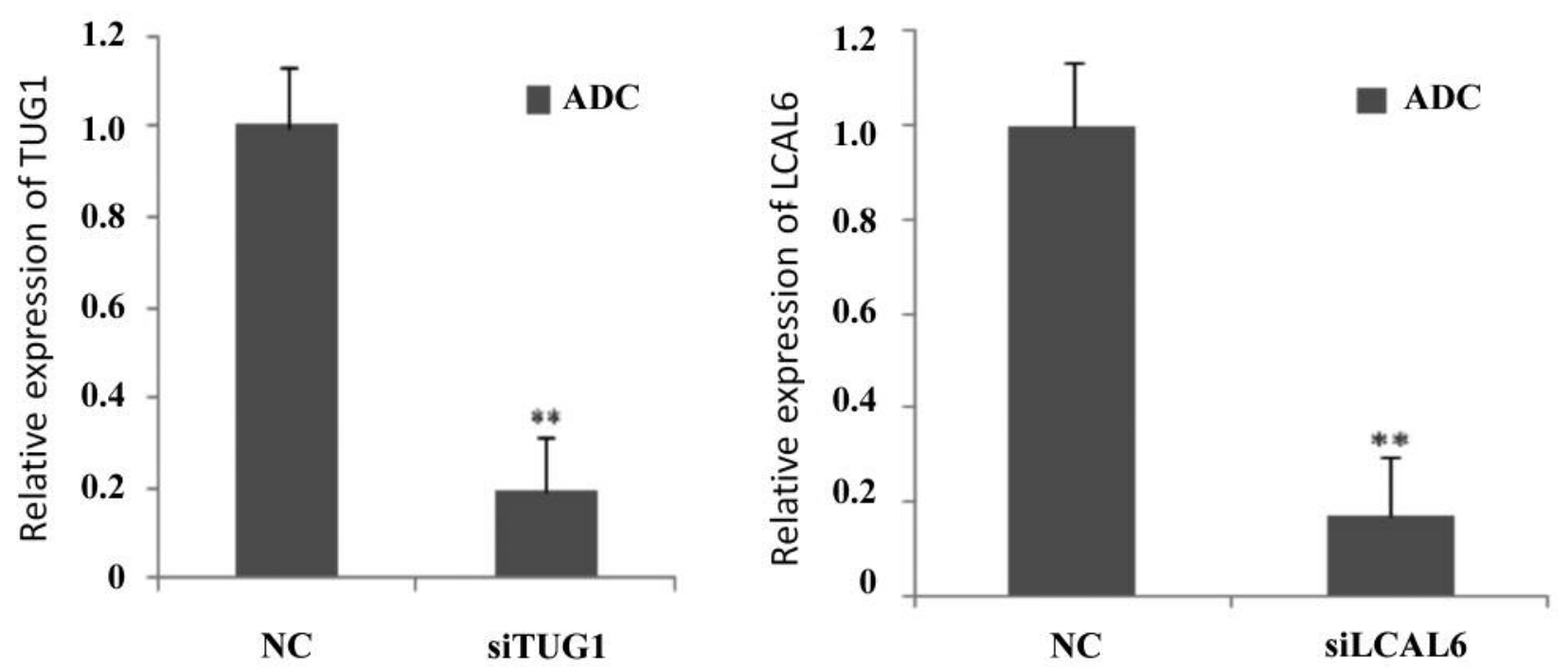

Figure 3. Effect of siTUG1 and siLCAL6 on expression of TUG1 and LCAL6, respectively, in NSCLC PDX models. Expression of TUG1 and LCAL6 in PDX tumors treated with siTUG1 and siLCAL6, respectively, was detected by RT-PCR. **p<0.01, when compared with normal control (NC). 
electrophoresis and electrophoretically transferred to a polyvinylidene fluoride membrane (Millipore, Bedford, MA, USA). The membranes were incubated with $5 \%$ nonfat milk solution for blocking non-specific binding and then with primary antibodies to Ki67 (1:300, VP-RM04, Vector Laboratories) HOXB7 (1:100, ab196007, Abcam, Cambridge, USA) and tubulin (Sigma, St. Louis, MO, USA. After washing twice with Tris buffered saline, the membranes were incubated with an appropriate secondary antibody (1:5000) conjugated with HRP for $2 \mathrm{~h}$ at room temperature. Analysis of electrochemiluminescence was performed according to the manufacturer's instructions using a Bio-Rad imaging system. Quantity One version software (Bio-Rad, Hercules, CA, USA) was used to quantify the density of bands.

Statistical analysis. The in vivo tumor take rates were compared with the $\mathrm{X}^{2}$ test. The significant difference between control and treated groups was analyzed using the Student's $t$-test. Analyses were performed using SPSS 20.0 statistical software. All statistical tests were two-sided with $p<0.05$ indicating statistical significance.

\section{Results and Discussion}

Establishment of NSCLC PDX nude mouse models. Twentysix NSCLC surgical specimens from primary tumors were subcutaneously implanted into immunodeficient mice leading to the establishment of 9 PDX models, representing a tumor take rate of $34.6 \%$.

To determine clinic-pathologic parameters related to PDX establishment success rates, tumor take rates were compared according to various patient characteristics. As shown in Table I, the tumor histological type was the only parameter that had a significant impact on the success rate of PDX establishment $\left(\mathrm{X}^{2}=3.884, p<0.05\right)$. Lung squamous cell carcinomas (SCC) were much more tumorigenic in nude mice $(7 / 12 ; 58.3 \%)$ compared to lung adenocarcinomas (ADC) $(2 / 11 ; 18.2 \%)$. Other factors, including age, sex, smoking status, tumor size, and overall tumor stage did not correlate with the success rate of PDX establishment $(p>0.05)$.

Preservation of parental NSCLC's biologic characteristics in $P D X$. The application of PDX models as a model system for human NSCLCs depends on the precise retention of the parental tumors' pathologic and molecular characteristics. As shown in Figure 1, histologic comparison revealed a high degree of similarity in the subtype-specific morphologic features and differentiation status between the parental tumors and corresponding xenografts. The expression of NSCLC histologic subtype-specific markers TTF-1, napsin A and p63 was compared by immunohistochemical analysis between patient tumors and corresponding PDXs. As shown in Table II, the expression of TTF-1 and napsin A protein in ADC patient tumors was not significantly changed in corresponding xenografts $(p>0.05)$. No significant difference in the expression level of p63 protein was found between SCC patient tumors and corresponding PDXs $(p>0.05)$.
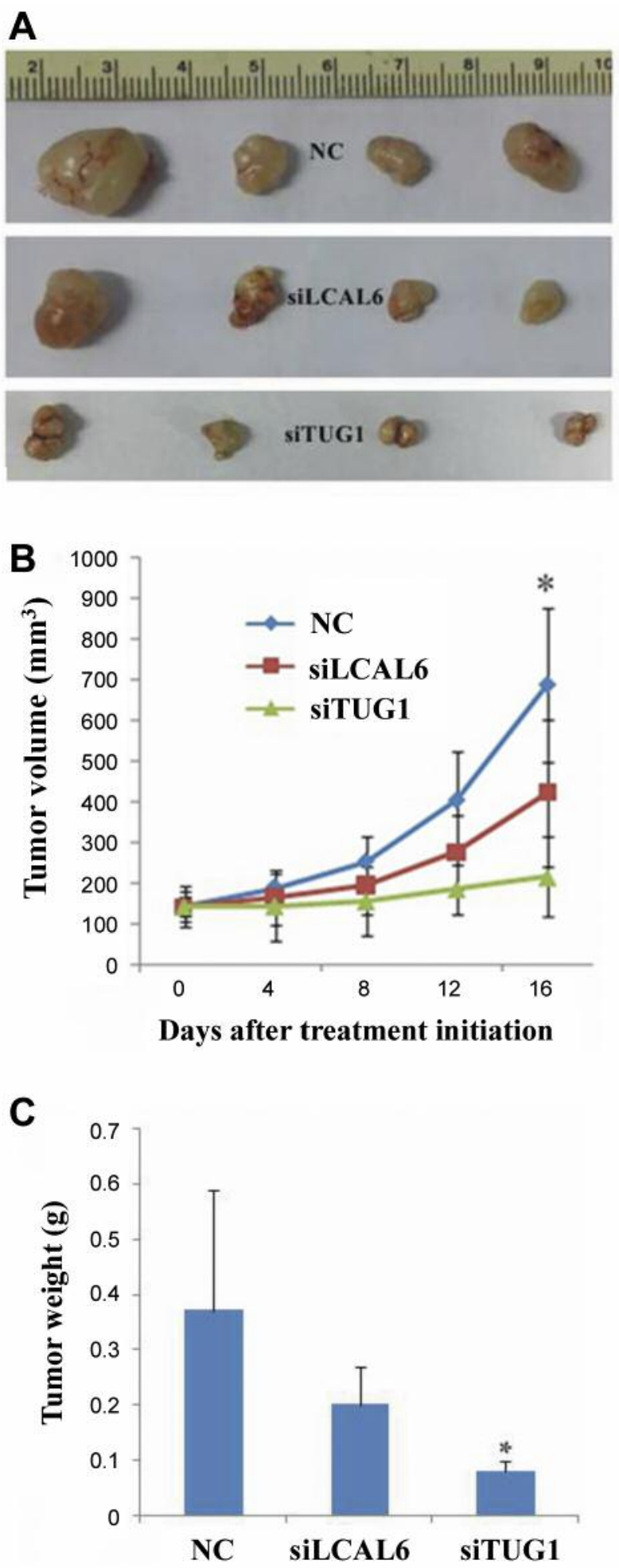

Figure 4. Effect of siTUG1 and siLCAL6 on tumor growth in NSCLC PDX models. Tumor-bearing mice were treated with siTUG1 and siLCAL6. Tumor growth was measured during the course of the experiment and final tumor weight was determined at autopsy. A. Tumor images in each group. B. Tumor growth curves of each group. C. Mean tumor weight in each group. ${ }^{*} p<0.05$, when compared with non-treated control (N.C.). 
A

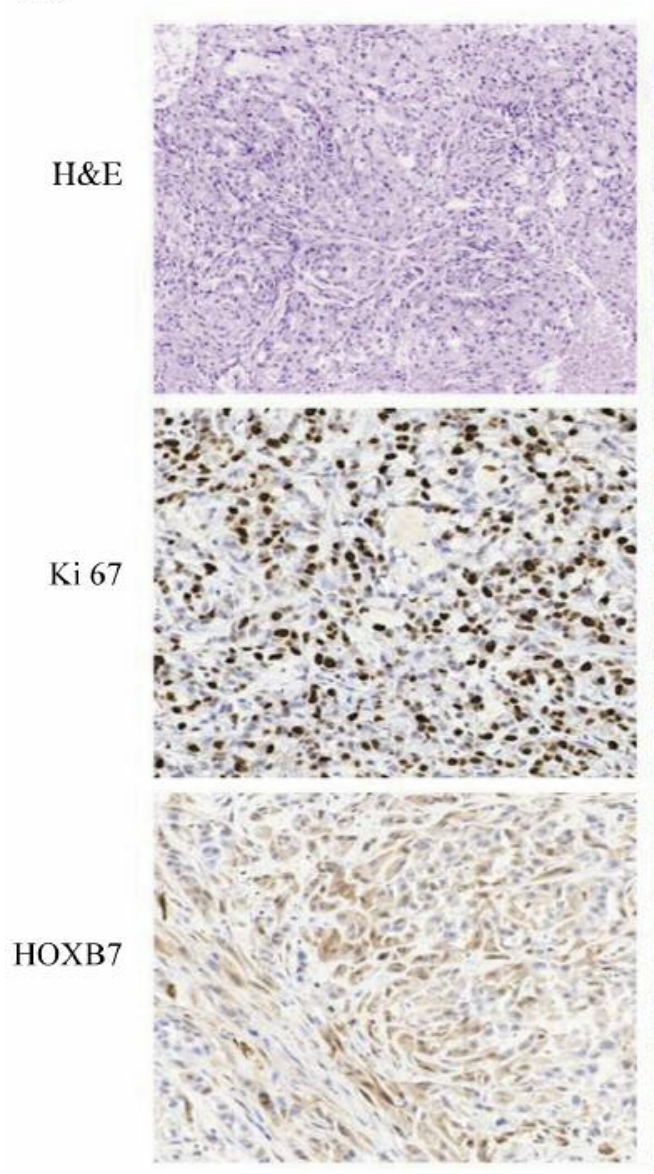

SiLCAL6
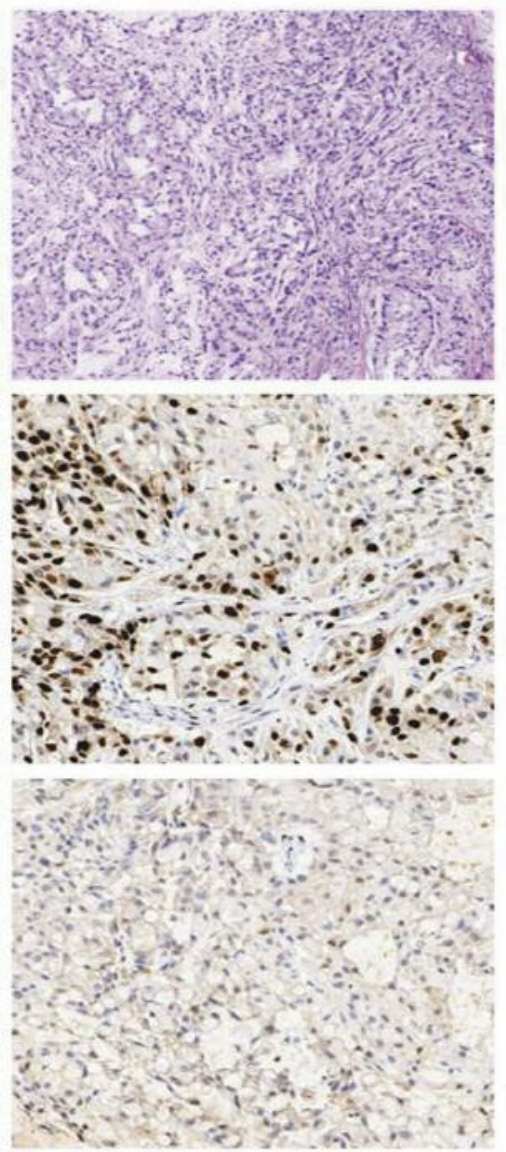

siTUG1
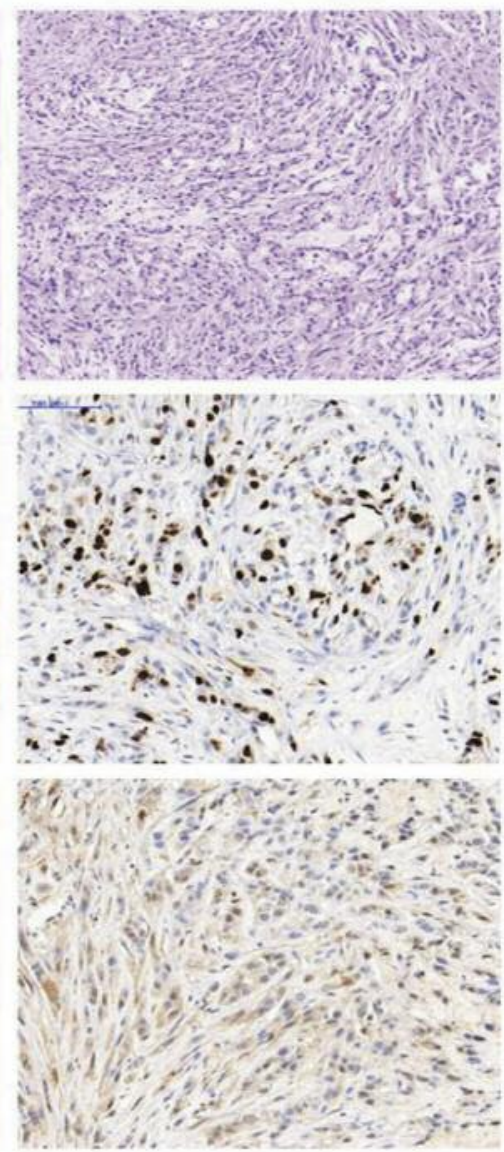

Moreover, we detected the expression level of TUG1 and LCAL6 in normal lung tissues, patient tumors and PDX tumors by RT-PCR. We found that both TUG1 and LCAL6 were strongly over-expressed in NSCLC patient tumors and PDXs as compared to normal lung tissues (Figure 2A). No significant change in expression level of TUG1 and LCAL6 was found between the patient tumors and corresponding PDX tumors (Figure 2B).

Effect of silencing of TUG1 and LCAL6 on expression of TUGland LCAL6 in the NSCLC PDX model. The expression of TUG1 and LCAL6 was detected using RT-PCR in the tumor tissues from the mice treated with siTUG1 and siLCAL6. As shown in Figure 3, the expression of TUG1 in the siTUG1treated group was significantly reduced compared to the control group $(p<0.01)$. The expression of LCAL6 in the siLCAL6treated group was also significantly lower than the control group $(p<0.01)$. This result indicated that the expression of TUG1 and LCAL6 in PDX models of NSCLC was suppressed by siTUG1 and siLCAL6 treatment, respectively.

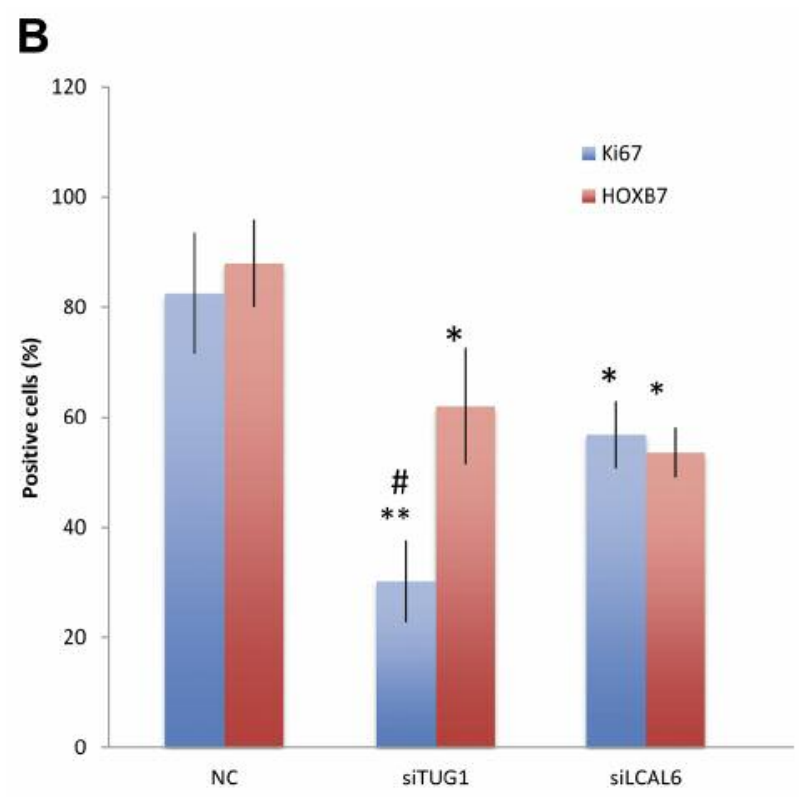

Figure 5. Continued 

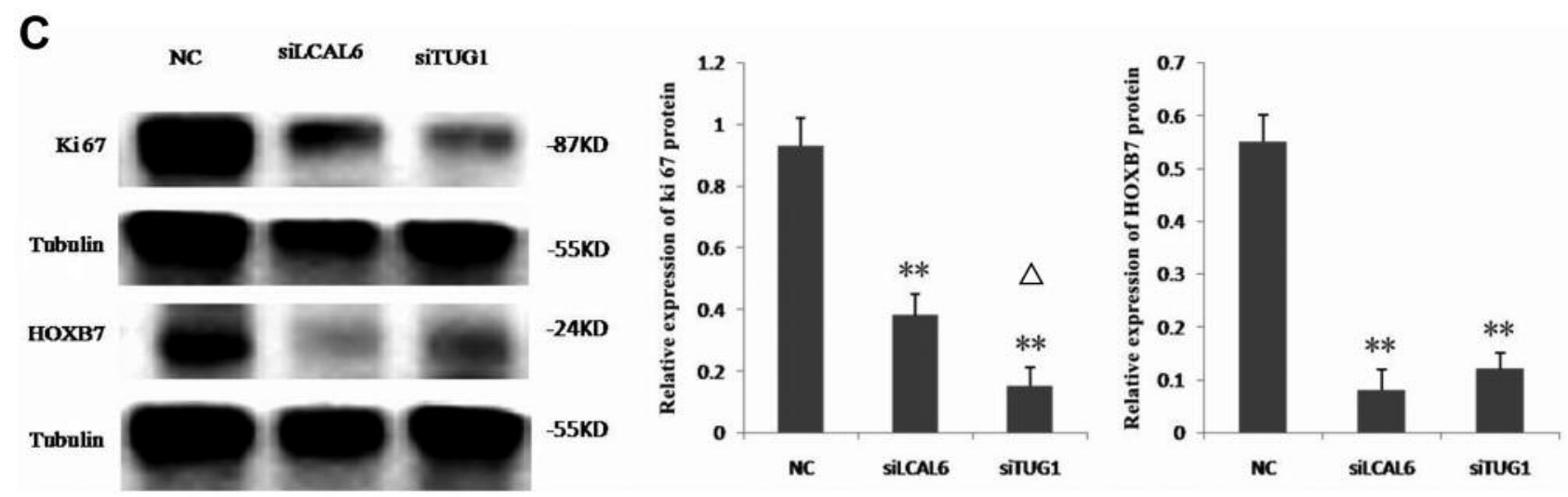

Figure 5. Effect of siTUG1 and siLCAL6 expression on Ki67 and HOXB7 in NSCLC PDX models. A. Representative images of H\&E staining and immunohistochemical analysis of Ki67 and HOXB7 expression in each group (200x magnification). B. Quantification for immunohistochemical staining for Ki67 and HOXB7 expression in each group. ${ }^{*} p<0.05$ or ${ }^{* * p}<0.01$, when compared with NC. \# $p<0.05$, when compared with siLCAL6 group. C. Western blotting analysis of Ki67 and HOXB7 expression in each group. ${ }^{* *} p<0.01$, when compared with NC. $\Delta=p<0.05$, when compared with siLCAL6 group.

Effect of silencing of TUG1 and LCAL6 on tumor growth in the NSCLC PDX model. The anti-tumor efficacy of siTUG1 and siLCAL6 was evaluated in a PDX model of lung adenocarcinoma. Tumor images, tumor growth curves and mean tumor weight in each group are shown in Figure 4. Tumor volume and weight were significantly reduced in the siTUG1-treated group compared to the control group $(p<0.05)$. However, no significant tumor-growth inhibition was found in the siLCAL6-treated group although lower mean tumor volume and weight was observed compared to the control group $(p>0.05)$.

Effect of silencing of TUG1 and LCAL6 on expression of Ki67 and HOXB7 in the NSCLC PDX model. Immunohistochemical staining and Western blotting were performed to assess the effects of siTUG1 and siLCAL6 treatment on protein expression of proliferation markers Ki67 and HOXgene-family HOXB7 in the tumors. As shown in Figures 5A and $\mathrm{B}$, immunohistochemical staining demonstrated positive protein expression of Ki67 and HOXB7 in the tumors of the control group. Ki67 expression was significantly suppressed in the siTUG1- and siLCAL6-treated groups compared to the control group $(p<0.01)$. The siTUG1 group showed more reduced Ki67 expression than the siLCAL6 group $(p<0.05)$. HOXB7 expression was significantly reduced in the siTUG1and siLCAL6-treated groups compared to the control group $(p<0.05)$. Western blotting analysis further confirmed significantly-reduced expression of Ki67 and HOXB7 protein in the siTUG1- and siLCAL6-treated groups compared to the control group $(p<0.01)$ (Figure 5C). As observed by immunohistochemical staining, the siTUG1 group also showed more reduced Ki67 expression than the
siLCAL6 group $(p<0.05)$. This result suggests siTUG1 can inhibit tumor Ki67 expression more than siLCAL6.

Numerous new long non-coding RNA (lncRNA) molecules have been shown to be involved in tumorigenesis and progression of (19). It was found that knockdown of TUG1 and LCAL6 inhibited cancer cell growth both in vitro and in vivo $(8,10)$.

In the present study, we explored the potential function of TUG1 and LCAL6 in NSCLCs by siRNA-mediated silencing in NSCLC PDX models. siTUG1 and siLCAL6 significantly reduced TUG1 and LCAL6 expression, respectively. However, only siTUG1 treatment resulted in significant tumor growth inhibition, suggesting possible greater therapeutic potential of targeting TUG1 than LCAL6 in NSCLC.

Ki67, a cell proliferation marker, was significantly suppressed by silencing of TUG1 and LCAL6 in the NSCLC PDX. The silencing with siTUG1 showed a more suppressive effect on Ki67 expression than siLCAL6, which may explain the greater anti-tumor efficacy of siTUG1 than siLCAL6 on the NSCLC PDX. Our result showed that HOXB7 expression was significantly reduced by silencing of TUG1 and LCAL6 in the NSCLC PDX model, indicating association of HOXgene with regulation of TUG1 and LCAL6.

PDX models are very important for the discovery and individualization of cancer therapy (20). The present study indicates that NSCLC histotype is most important in establishment of PDX models with squamous cell carcinoma having a greater establishment rate than adenocarcinoma. Our studies in other cancer types have suggestd that tumor size (21) and tumor grade and therapeutic pretreatment (16), are very important for establishment. 
Future experiments will focus on patient-derived orthotopic xenograft (PDOX) of NSCLC (13) in order to study metastasis and its therapeutic inhibition.

\section{Conflicts of Interest}

None of the Authors have a conflict of interest in regard to this study.

\section{Acknowledgements}

This research was supported by the Research Project of Jinling Hospital (No. 2016044).

\section{References}

1 Spira A and Ettinger DS: Multidisciplinary management of lung cancer. N Engl J Med 350: 379-392, 2004.

2 Hoffman PC, Mauer AM and Vokes EE: Lung cancer. Lancet 355: 479-485, 2000.

3 Cappuzzo F, Ciuleanu T, Stelmakh L, Cicenas S, Szczesna A and Juhasz E: Erlotinib as maintenance treatment in advanced nonsmall-cell lung cancer: a multicentre, randomised, placebocontrolled phase 3 study. Lancet Oncol 11: 521-529, 2010.

4 Fukuoka M, Wu YL, Thongprasert S, Sunpaweravong P, Leong SS and Sriuranpong V: Biomarker analyses and final overall survival results from a phase III, randomized, open-label, firstline study of gefitinib versus carboplatin/paclitaxel in clinically selected patients with advanced non-small-cell lung cancer in Asia (IPASS). J Clin Oncol 29: 2866-2874, 2011.

5 Shaw AT, Kim DW, Mehra R, Tan DS, Felip E, Chow LQ, Camidge DR, Vansteenkiste J, Sharma S, De Pas T, Riely GJ, Solomon BJ, Wolf J, Thomas M, Schuler M, Liu G, Santoro A, Lau YY, Goldwasser M, Boral AL and Engelman JA: Ceritinib in ALK-rearranged non-small-cell lung cancer. N Engl J Med 370: 1189-1197, 2014.

6 Khalil AM, Guttman M and Huarte M: Many human large intergenic noncoding RNAs associate with chromatin-modifying complexes and affect gene expression. Proc Natl Acad Sci USA 106: 11667-11672, 2009.

7 Yang L, Lin C and Liu W: IncRNA- and Pc2 methylationdependent gene relocation between nuclear structures mediates gene activation programs. Cell 147: 773-788, 2011.

8 Zhang Q, Geng PL, Yin P,Wang XL, Jia JP and Yao J: Downregulation of long non-coding RNA TUG1 inhibits osteosarcoma cell proliferation and promotes apoptosis. Asian Pac J Cancer Prev 14: 2311-2315, 2013.

9 Han Y, Liu Y, Gui Y and Cai Z: Long intergenic non-coding RNATUG1 is overexpressed in urothelial carcinoma of the bladder. J Surg Oncol 107: 555-559, 2013.

10 Qiu MT, Feng DJ, Zhang HT, Xia W, Xu Y, Wang J, Dong G, Zhang Y, Yin R and Xu L: Comprehensive analysis of lncRNA expression profiles and identification of functional lncRNAs in lung adenocarcinoma. Oncotarget 7: 16012-16022, 2016.
11 Fan S, Yang Z, Ke Z, Huang K, Liu N, Fang X and Wang K: Down-regulation of the long non-coding RNA TUG1 is associated with cell proliferation, migration, and invasion in breast cancer. Biomed Pharmacother 95: 1636-1643, 2017.

12 Hoffman RM, ed: Patient-Derived Mouse Models of Cancer. Molecular and Translational Medicine. Series eds., Coleman WB, Tsongalis GJ. ISSN: 2197-7852, 2017.

13 Wang X, Fu X and Hoffman RM: A new patient-like metastatic model of human lung cancer constructed orthotopically with intact tissue via thoracotomy in immunodeficient mice. Int $\mathrm{J}$ Cancer 51: 992-995, 1992.

14 Wang X, Fu X, Kubota T and Hoffman RM: A new patient-like metastatic model of human small-cell lung cancer constructed orthotopically with intact tissue via thoracotomy in nude mice. Anticancer Res 12: 1403-1406, 1992.

15 Wang X, Fu X and Hoffman RM: A patient-like metastasizing model of human lung adenocarcinoma constructed via thoracotomy in nude mice. Anticancer Res 12: 1399-1402, 1992.

16 Russell TA, Eckardt M, Murakami T, Elliott I, Kawaguchi K, Kiyuna T, Igarashi K, Li Y, Crompton J, Graham DS, Dry SM, Bernthal N, Yanagawa J, Kalbasi A, Federman M, Chmielowski B, Singh A, Hoffman RM and Eilber FC: Clinical factors that affect the establishment of soft tissue sarcoma patient-derived orthotopic xenografts: A University of California, Los Angeles, sarcoma program prospective clinical trial. JCO Precision Oncol, 2017. doi: https://doi.org/10.1200/PO.17.00071 [Epub ahead of print]

17 Zhang XC, Zhang J, Li M, Huang XS, Yang XN, Zhong WZ, Xie L, Zhang L, Zhou M, Gavine P, Su X, Zheng L, Zhu G, Zhan P, Ji Q and Wu YL: Establishment of patient-derived nonsmall cell lung cancer xenograft models with genetic aberrations within EGFR, KRAS and FGFR1: useful tools for preclinical studies of targeted therapies. J Transl Med 11: 168-178, 2013.

18 John T, Kohler D, Pintilie M, Yanagawa N, Pham NA, Li M, Panchal D, Hui F, Meng F, Shepherd FA and Tsao MS: The ability to form primary tumor xenografts is predictive of increased risk of disease recurrence in early-stage non-small cell lung cancer. Clin Cancer Res 17: 134-141, 2011.

19 Nakagawa T, Endo H, Yokoyama M, Abe J, Tamai K, Tanaka N, Sato I, Takahashi S, Kondo T and Satoh K: Large noncoding RNA HOTAIR enhances aggressive biological behavior and is associated with short disease-free survival in human non-small cell lung cancer. Biochem Biophys Res Commun 436: 319-324, 2013.

20 Hoffman RM, ed. In: Patient-Derived Mouse Models of Cancer. Hoffman, R.M., ed. Molecular and Translational Medicine. Series eds., Coleman, WB, Tsongalis, GJ ISSN: 2197-7852, 2017.

21 Jun E, Jung J, Jeong S-Y, Choi EK, Kim MB, Lee JS, Hong SM, Seol H, S, Hwang C, Hoffman RM, Shim IK, Chang S and Kim SC: Surgical and oncological factors affecting the successful engraftment of patient-derived xenografts in pancreatic ductal adenocarcinoma. Anticancer Res 36: 517-522, 2016.

Received October 10, 2017

Revised October 31, 2017

Accepted November 1, 2017 